\title{
DEVELOPMENT OF ANALYTICAL MODEL AND NUMERICAL ANALYSIS FOR SPIRAL COIL TYPE GROUND HEAT EXCHANGERS
}

\author{
S. K. Park ${ }^{1}$, S. R. Lee ${ }^{2}$, S. Yoon ${ }^{3}$, G. H. Go ${ }^{4}$ \\ ${ }^{1}$ Department of Civil and Environmental Engineering, KAIST, Daejeon, Republic of KOREA \\ ${ }^{2}$ Department of Civil and Environmental Engineering, KAIST, Daejeon, Republic of KOREA \\ (srlee@kaist.ac.kr) \\ ${ }^{3}$ Department of Civil and Environmental Engineering, KAIST, Daejeon, Republic of KOREA \\ ${ }^{4}$ Department of Civil and Environmental Engineering, KAIST, Daejeon, Republic of KOREA
}

\begin{abstract}
A spiral coil source model and its analytical solution was developed to consider 3dimensional shape effects and radial dimension effects of a spiral coil type ground heat exchanger (GHE) using Green's function method. The spiral coil source model was transformed into a formula of error function to improve and simplify computation for the engineering application. To analyze the characteristics of the analytical model, the analytical model's prediction results were compared with numerical analysis results implemented in ABAQUS/Standard.
\end{abstract}

Keywords: Spiral coil type GHE, spiral coil source model, analytical model, numerical analysis.

\section{INTRODUCTION}

Interest in renewable energy has gradually increased over the world. In particular, ground-coupled heat pump (GCHP) systems have been applied to obtain shallow-depth geothermal energy with satisfactory energy efficiency. The GCHP uses sustainable ground temperature to emit heat during the summer and vice versa during the winter for cooling and heating buildings.

However, installation of a GCHP system involves additional costs for boring. To overcome this, the geothermal energy pile has been developed as efficient means of reducing the installation cost. Furthermore, coil type heat exchangers are recently being used in the energy pile in order to obtain high heat exchange efficiency. The coil type heat exchanger has more complex geometry than the U-tube but engineers still used commercial design programs based on one-dimensional analytical solutions without considering the complex geometry. 
In engineering fields, regardless of the limits by the process of simplifying assumptions, analytical solutions with simplicity and convenience of computation are used. Representatively, classical models for GHE are the line source model (Carslaw and Jaeger, 1947) and the cylindrical source model (Ingersoll and Zobel, 1954). However, these classical models are not adequate for a coil type heat exchanger. Recently, the models for spiral coil type GHE were developed as the followed; a new solid cylindrical source model (Man et al., 2010), a ring coil source model (Cui et al., 2011), and a spiral heat source model (Man et al, 2011). Although the coil models were developed, those are not exact solutions for spiral coil type sources and have a problem of limitation in calculating convergent results, with complicated formulas. Thus, research is necessary in order to obtain a simple exact analytical model of a coil type heat exchanger.

This paper describes the development of an efficient spiral coil source model. Its analytical solution was developed to consider 3-dimensional shape effects and radial dimension effects of a spiral coil type GHE using Green's function method. The spiral coil source model was transformed into a formula of error function to improve and simplify computation for the engineering application. Also, this paper analyzed the characteristics of the analytical model through the analytical model's prediction results compared with numerical analysis results implemented in ABAQUS/Standard.

\section{ANALYTICAL MODELS}

A widely used model is the infinite line source model. The model, developed and evaluated by Carslaw and Jaeger (1947), applies Kelvin's heat source theory to ground heat exchangers. Ingersoll and Plass (1948) provided tabulated values of the evaluated integral solution part. In addition, simple correlations have been proposed to approximate the integral by Bose (1991).

Another widely used model is the infinite cylindrical source model. The model was mentioned by Carslaw and Jaeger (1947), but later proposed by Ingersoll and Zobel (1954). The finite line source model was developed by Eskilson (1987). These classical models, however, result in imprecise design of the GHE pile, because of limitations due to simplifying assumptions of a heat source.

Recently, Lamarche and Beauchamp (2007) presented an improved solution of the finite cylindrical source model. Lee and Lam (2007) suggested a 3-dimensional numerical model for a GHE pile. Man et al. (2010) presented a new solid cylindrical source model for a 1-D and 2-D coil type GHE. Cui et al. (2011) also developed a transient ring-coil source model for a 1-D and 2-D coil type GHE. Man et al. (2011) developed a spiral heat source model.

\section{NUMERICAL ANALYSIS}

The thermal response tests (Yoon et al., 2011) were numerically simulated using conductive heat transfer analysis scheme implemented in ABAQUS/Standard. The model chamber was filled with typical Korean sand named Joomoonjin sand in dry condition, as shown in 
Fig. 1 and Table 1). Based on the sand-raining method, nearly homogeneous sand ground was made up in the chamber.

A coil type HDPE pipe (pipe diameter $=25 \mathrm{~mm}$, spacing of coils $=5 \mathrm{~cm}$ ) with coil diameter of $25 \mathrm{~cm}$ and coil length of $4 \mathrm{~m}$ were installed horizontally in the soil. Two temperature sensors were installed at inlet and outlet of GHE pipe. RTD sensors were also attached at two locations ( $25 \mathrm{~cm}$ and $35 \mathrm{~cm}$ from the center and $1.8 \mathrm{~m}$ from the top).

The coil heat exchanger was simply modeled as heat source emitting a constant temperature. The spiral coil configuration of heat sources was considered in the analyses. The 2dimensional analyses for the spiral coil cases were applied to model the coil heat exchanger as shown in Fig. 2. A half of the configuration of vertical center of GHE is considered because of the axisymmetric condition.
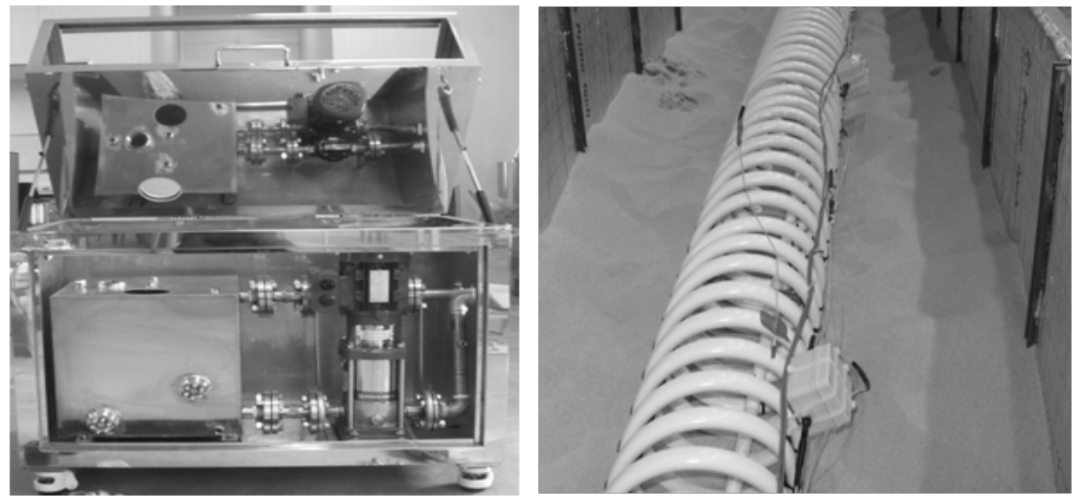

Figure 1. Thermal response test equipment (left) and coil type GHE (right).

Table 1. Physical and thermal properties of Joomoonjin sand

\begin{tabular}{l|c}
\multicolumn{1}{c|}{ Parameter } & Value \\
\hline Unit dry density $\gamma\left(\mathrm{kN} / \mathrm{m}^{3}\right)$ & 1397 \\
\hline Void ratio $e$ & 0.9 \\
\hline Thermal conductivity $\lambda(\mathrm{W} / \mathrm{m})$ & 0.28 \\
\hline Specific heat capacity $c(\mathrm{~J} / \mathrm{kg} \mathrm{K})$ & 800 \\
\hline
\end{tabular}

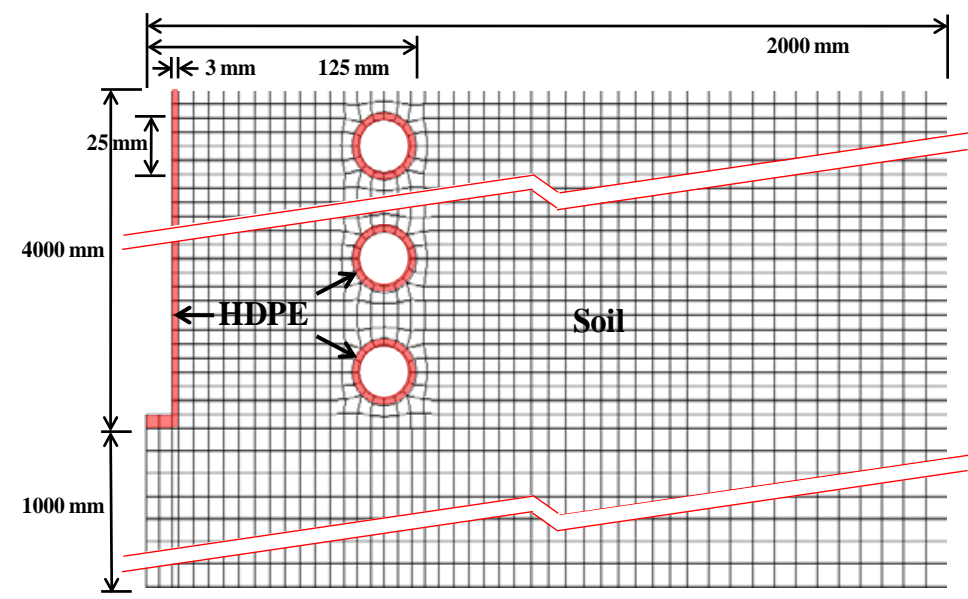

Figure 2. Schematic view of numerical model for simulation of TRT. 


\section{SPIRAL COIL SOURCE MODEL AND ITS SOLUTION}

Because of the complex geometry of the spiral coil type GHE, an efficient spiral coil source model and its analytical solution have been developed. In the model, the 3-dimensional shape effects and radial dimension effects of a spiral coil type GHE were considered using Green's function.

A spiral coil type GHE is buried in a pile, as shown in Fig. 3. For the spiral coil type GHE, the coil radius is $r_{0}$, the coil depth is $h$, and the number of coil turns is $N$, respectively. For modeling the heat transfer from the buried spiral coil type GHE, the following assumptions were made:

(1) The ground is assumed to be a homogeneous infinite medium. The thermal properties of the ground do not change with the variation of temperature.

(2) The spiral coil heat source is assumed to take a spiral coil form without a vertical outlet pipe. The spiral coil heat source is buried in the ground from the ground surface to a certain depth, h. Mass, heat capacity and thickness of the heat source are neglected. The heating rate per length of pile, q, is constant from the starting instant, $\mathrm{t}=0$.

(3) The medium has a uniform initial temperature, $t_{0}$.

(4) The ground surface, the boundary of the medium, $z=0$, maintains a constant temperature.

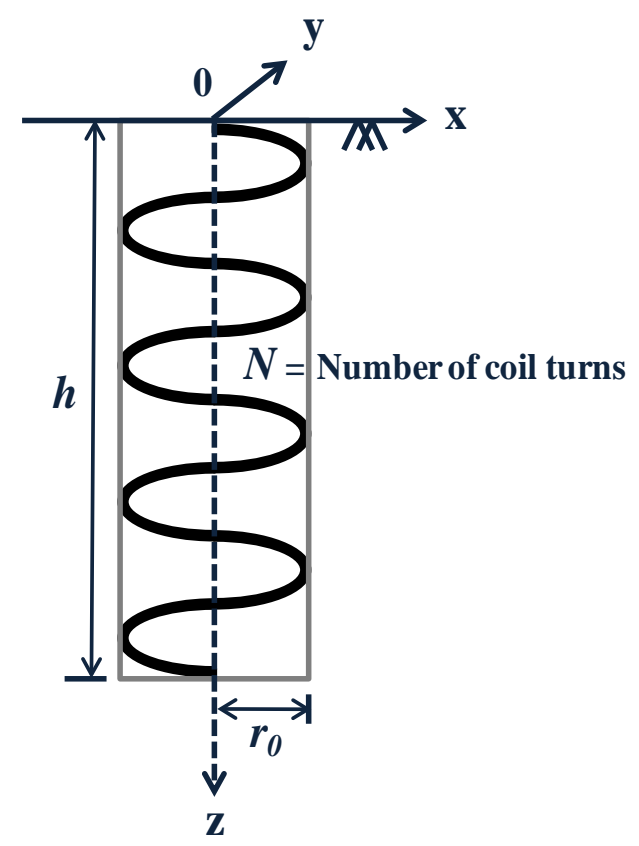

Figure 3. The spiral coil source.

The transient 3-D conduction problem based on the governing equation of the transient heat conduction along with the given boundary and initial conditions in the infinite medium can then be formulated in the Cartesian coordinates: 


$$
\begin{aligned}
& \frac{\partial \theta}{\partial t}=\alpha \Delta \theta+Q(u, t) \quad \text { for } \quad z>0 \\
& Q(u, t)=\frac{q_{l}}{\rho c} \delta(u-s(z)) \quad \text { for } \quad 0 \leq z \leq h . \\
& \theta(u, 0) \equiv 0 \quad \text { for } \quad z>0 \\
& \theta(u, t)=0 \quad \text { for } z=0
\end{aligned}
$$

where the wave number is $\omega=2 N \pi / h, \delta(u-s(z))$ is the Dirac $\delta$-function, and the heat source is $s(z)=\left(r_{0} \cos (\omega z), r_{0} \sin (\omega z), z\right)$.

The spiral coil heat source $\mathrm{Q}(\mathrm{u}, \mathrm{t})$ can be formulated according to Green's function method. Then, for modeling a semi-infinite interval $\mathrm{z}>0$, a virtual spiral coil heat source with the same heat rate $\mathrm{q}_{1}$ and negative depth $-\mathrm{h}$ is assumed on a symmetric space to the boundary. An analytical solution of spiral coil source problem can then be derived as Eq. (2).

$$
\begin{aligned}
\theta(u, t) & =\frac{q_{l}}{\rho c} \int_{0}^{t} \int_{0}^{\infty} \tilde{G}\left(u, t ; x^{\prime}=r_{0} \cos \left(\omega z^{\prime}\right), y^{\prime}=r_{0} \sin \left(\omega z^{\prime}\right), z^{\prime}, t^{\prime}\right) d z^{\prime} d t^{\prime} \\
& =\frac{q_{l}}{(4 \pi \alpha)^{3 / 2} \rho c} \int_{0}^{t} \frac{1}{\left(t-t^{\prime}\right)^{3 / 2}} \int_{0}^{h} e^{-\frac{F\left(x, y, z^{\prime}\right)}{4 \alpha\left(t-t^{\prime}\right)}}\left(e^{-\frac{\left(z-z^{\prime}\right)^{2}}{4 \alpha\left(t-t^{\prime}\right)}}-e^{-\frac{\left(z+z^{\prime}\right)^{2}}{4 \alpha\left(t-t^{\prime}\right)}}\right) d z^{\prime} d t^{\prime}
\end{aligned}
$$

with

$$
F\left(x, y, z^{\prime}\right)=x^{2}+y^{2}+r_{0}^{2}-2 x r_{0} \cos \left(\omega z^{\prime}\right)-2 y r_{0} \sin \left(\omega z^{\prime}\right)
$$

For computational purpose, using the error function by changing the variable $\xi^{2}=A /\left(4 \alpha\left(t-t^{\prime}\right)\right)$, Eq. (2) can be expressed by a simple form of Eq. (4).

$$
\theta(u, t)=\frac{q_{l}}{4 \pi \lambda} \int_{0}^{h} \frac{\operatorname{erfc}\left(A_{-}\left(u, z^{\prime}\right) / 2 \sqrt{\alpha t}\right)}{A_{-}\left(u, z^{\prime}\right)}-\frac{\operatorname{erfc}\left(A_{+}\left(u, z^{\prime}\right) / 2 \sqrt{\alpha t}\right)}{A_{+}\left(u, z^{\prime}\right)} d z^{\prime} .
$$

with

$$
A_{ \pm}\left(u, z^{\prime}\right)=\sqrt{F\left(x, y, z^{\prime}\right)+\left(z \pm z^{\prime}\right)^{2}} .
$$

\section{VERIFICATION AND COMPARISON}

Fig. 4 shows the comparisons of test results and predictions. The results show reasonable agreements between the analysis results and the analytical solution of spiral coil source model. However, the analytical solution of line source model estimates lower soil temperature rise compared to the others. On the contrary, the analytical solution of cylindrical source model estimates greater soil temperature rise compared to the others. 


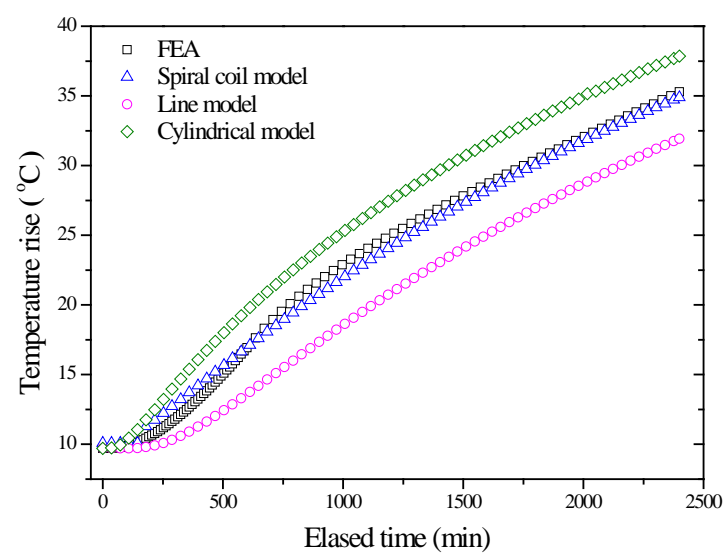

(a) Spacing $=5 \mathrm{~cm}$, measured point $=25 \mathrm{~cm}$

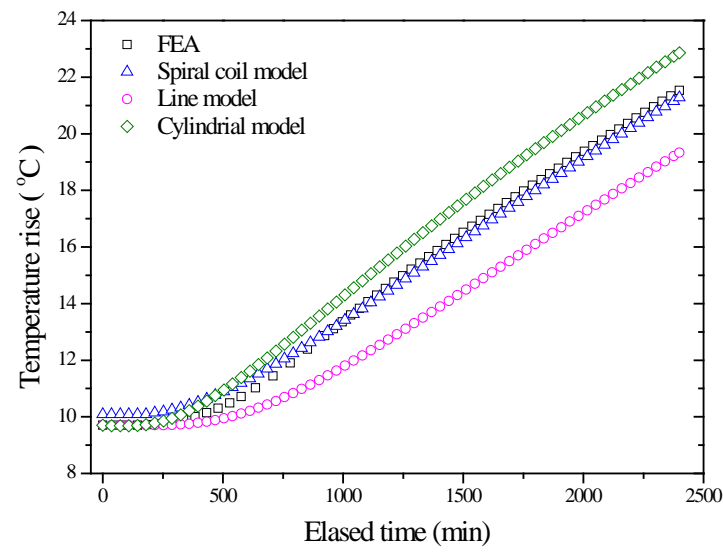

(b) Spacing $=5 \mathrm{~cm}$, measured point $=35 \mathrm{~cm}$

Figure 4. Comparisons of the measured soil temperatures and predictions.

\section{DISCUSSION}

\subsection{Temperature distribution of various spiral coil sources}

Fig. 5 shows characteristics of spiral coil source model. The results show comparison of temperature distribution of various spiral coil sources. Because of a spiral form along the depth, Figs. 5a, 5b and 5c show a fluctuation of temperature. The bigger a spacing of spiral coil source is, the more rough a fluctuation of temperature is, as shown in fig. 5c.

\subsection{Special case of the analytical solution}

When a spiral coil becomes a line, that means the radius of coil $r_{0}$ is zero, the solution of spiral coil source model reduces to the finite line source model (Eskilson, 1987) because of $\mathrm{F}\left(\mathrm{x}, \mathrm{y}, \mathrm{z}^{\prime}\right)=\mathrm{x}^{2}+\mathrm{y}^{2}=\mathrm{r}^{2}$ in cylindrical coordinates. 


$$
\begin{aligned}
\theta(u, t) & =\frac{q_{l}}{4 \pi \lambda} \int_{0}^{h} \frac{\operatorname{erfc}\left(\sqrt{x^{2}+y^{2}+\left(z-z^{\prime}\right)^{2}} / 2 \sqrt{\alpha t}\right)}{\sqrt{x^{2}+y^{2}+\left(z-z^{\prime}\right)^{2}}}-\frac{\operatorname{erfc}\left(\sqrt{x^{2}+y^{2}+\left(z+z^{\prime}\right)^{2}} / 2 \sqrt{\alpha t}\right)}{\sqrt{x^{2}+y^{2}+\left(z+z^{\prime}\right)^{2}}} d z^{\prime} \\
& =\frac{q_{l}}{4 \pi \lambda} \int_{0}^{h} \frac{\operatorname{erfc}\left(\sqrt{r^{2}+\left(z-z^{\prime}\right)^{2}} / 2 \sqrt{\alpha t}\right)}{\sqrt{r^{2}+\left(z-z^{\prime}\right)^{2}}}-\frac{\operatorname{erfc}\left(\sqrt{r^{2}+\left(z+z^{\prime}\right)^{2}} / 2 \sqrt{\alpha t}\right)}{\sqrt{r^{2}+\left(z+z^{\prime}\right)^{2}}} d z^{\prime}
\end{aligned}
$$

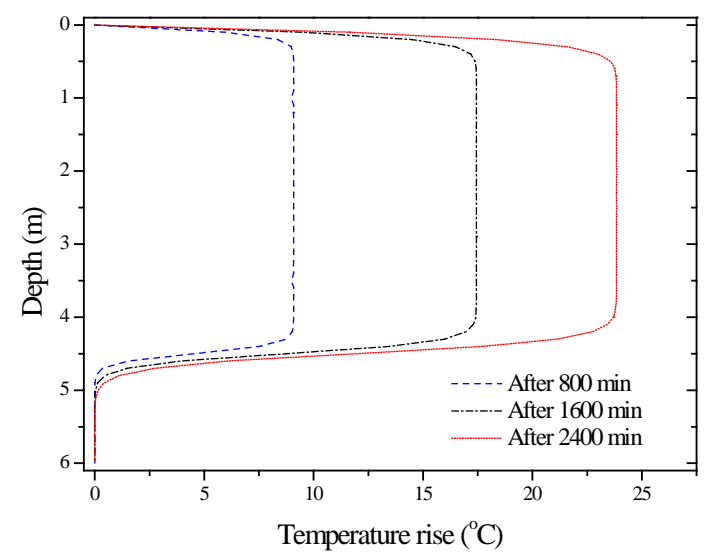

(a) Spacing $=5 \mathrm{~cm}$

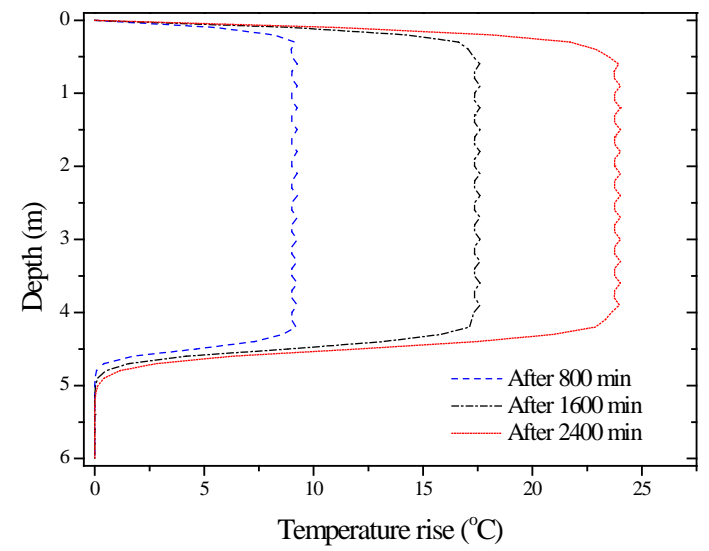

(b) Spacing $=15 \mathrm{~cm}$

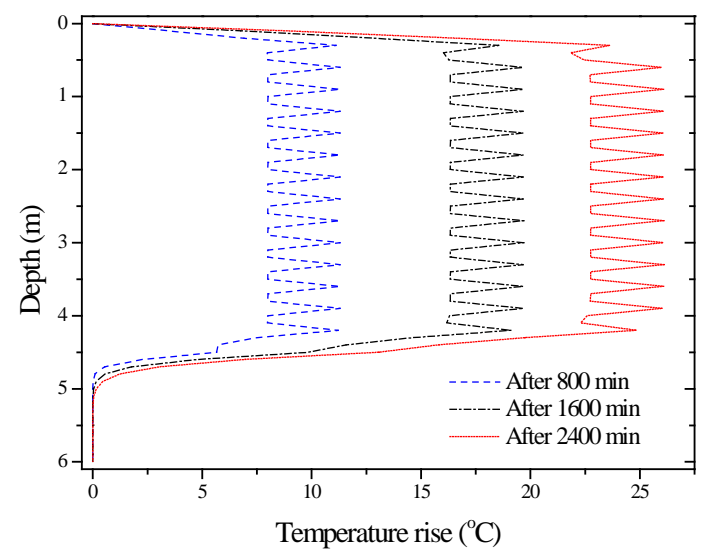

(c) Spacing $=25 \mathrm{~cm}$

Figure 5. Comparison of temperature distributions of various spiral coil source. 


\section{CONCLUSIONS}

In this study, an efficient spiral coil source model and its analytical solution were developed. Characteristics were analyzed and prediction results were compared with numerical analysis results implemented in ABAQUS/Standard. According to the research results, the following conclusion can be deduced.

(1) The results of analytical solution of spiral coil source model are in good agreement with the overall behavior. The line source model predicts the underestimated results and the cylindrical source model predicts the overestimated results for the spiral coil GHE. The efficient analytical model seems to be an adequate prediction tool for the spiral coil GHE.

(2) The spiral coil source model presents properly a 3-D effect caused by the spiral form. When the radius of coil r0 becomes zero, the solution of spiral coil source model reduces to Eskilson's finite line source model.

\section{ACKNOWLEDGEMENTS}

This research was supported by the Korea Electric Power Research Institute of the Korea Electric Power Corporation and the 2011 Construction Technology Innovation Project (11 Technology Innovation E04) by the Korea Institute of Construction and Transportation Technology Evaluation and Planning funded by the Ministry of Land, Transport, and Maritime Affair.

\section{REFERENCES}

[1] ABAQUS Inc., ABAQUS user's manual Ver. 6.5. Rhode Island, 2004.

[2] Bose, J., Design and Installations Standards. International Ground Source Heat Pump Association. Stillwater, 1991.

[3] Carslaw, H. and Jaeger, J., Conduction of Heat in Solids. Oxford, 1947.

[4] Cui, P., Li, X., Man, Y. and Fang, Z., "Heat transfer analysis of pile geothermal heat exchangers with spiral coils”. Applied Energy. 88. 4113-4119, 2011.

[5] Eskilson, Thermal Analysis of Heat Extraction Boreholes. Ph.D. Thesis. University of Lund. Sweden, 1987.

[6] Ingersoll, L. and Plass, H., "Theory of the ground pipe heat source for the heat pump". Piping and Ari conditioning. 20. 119-122, 1948.

[7] Ingersoll, L., Zobel., O and Ingersoll, A., Heat Conduction with Engineering Geological and other Applications. McGraw-Hill. New York, 1954.

[8] Lamarche, L. and Beauchamp, B., "A new contribution to the finite line-source model for geothermal boreholes”. Energy and Buildings. 39. 188-198, 2007.

[9] Lee, K. and Lam, H., "Computer simulation of borehole ground heat exchangers for geothermal heat pump systems”. Renewable Energy. 33. 1286-1296, 2007.

[10] Man, Y., Yang, H., Diao, N., Liu, J. and Fang, Z., “A new model and analytical solutions for borehole and pile ground heat exchangers". International Journal of Heat and 
Mass Transfer. 53. 2593-2601, 2010.

[11] Man, Y., Yang, H., Diao, N., Cji, P., Liu, L. and Fang, Z., "Development of spiral heat source model for novel pile ground heat exchangers". HVAC and R Research. 17. 6. 1075-1088, 2011.

[12] Yoon, S., Park, S. K., Park, H. K., Go, G. H. and Lee, S. R., "Evaluation of heat transfer characteristics in double-layered and single-layered soils”. Korea Society of Geothermal Energy Engineers. 7. 2. 43-50, 2011 (in Korean). 